\title{
EL PROCEDIMIENTO AGRAVADO DE REFORMA DE LA CONSTITUCIÓN DE 1978
}

\author{
POR \\ PIEDAD GARCÍA-ESCUDERO MÁRQUEZ \\ Profesora Titular de Derecho Constitucional \\ Universidad Complutense de Madrid
}

1. El anuncio por el Presidente del Gobierno en su discurso de investidura de su propósito de poner en marcha la reforma de la Constitución ha generado una numerosa bibliografía sobre las cuatro cuestiones afectadas por la reforma. Sin embargo, llama la atención el escaso interés prestado a la tramitación que deberá seguir la reforma, pese a las múltiples cuestiones que aquélla suscita, en particular cuando ha de sujetarse al procedimiento agravado previsto en el artículo 168 de la Constitución.

El propio Informe del Consejo de Estado sobre la reforma constitucional dedica apenas 17 de las 383 páginas que lo integran al procedimiento de reforma. Y, sin embargo, en estas pocas páginas se plantean problemas varios, tales como: procedimiento único o múltiple, proyectos o proposiciones de reforma, qué debe entenderse por principio de reforma, mayoría de aprobación del principio por las segundas Cortes, momento de elaboración del texto, eventual solución de los conflictos entre Cámaras... Estos y otros problemas, supuestamente desarrollados y resueltos en tan breve espacio, considero que pueden ser objeto de un tratamiento más detenido, desde un punto de vista estrictamente jurídico, objetivo al que he dedicado una monografía ${ }^{1}$, de la que este trabajo constituye una síntesis.

${ }^{1}$ P. García-Escudero MárQuez, El procedimiento agravado de reforma de la Constitución de 1978, Centro de Estudios Políticos y Constitucionales, Madrid, 2007. 
Aunque existan ya estudios sobre la materia, la supuesta inminencia de una reforma constitucional, la inclusión de un apartado dedicado al procedimiento en el citado Informe del Consejo de Estado y las reflexiones contenidas en el mismo proporcionan la ocasión para un reexamen de la cuestión. Por otra parte, la existencia de un precedente de tramitación de una reforma ordinaria de la Constitución conforme al artículo 167 de la misma me ha movido a estudiar el procedimiento aún inédito contenido en el artículo 168 de la Norma fundamental. Si, en ocasiones, más allá de la crítica de este precepto, la doctrina se ha centrado en el examen y comentario exhaustivo del artículo 167, considerándolo uno de los artículos más importantes de la Constitución, ha sido por entender que la extraordinaria complejidad del procedimiento previsto en el artículo 168 lo hace difícilmente transitable y convierte al primero en el procedimiento de reforma operativo, practicable y, por supuesto, ordinario o normal.

2. La importancia del procedimiento puede subrayarse recordando la distinción de Schmitt entre reformas constitucionales e inconstitucionales de la Constitución, verificadas con o sin observación del procedimiento constitucionalmente prescrito para tales reformas, de forma que sólo las primeras son reformas o revisiones constitucionales en sentido propio. Entre nosotros, cabe citar la declaración de Peces $\mathrm{Barba}^{2}$ de que el primer y más evidente criterio formal en relación con la reforma constitucional es que debe hacerse de acuerdo con los procedimientos establecidos en la propia Constitución en su Título X: «si estas formas no se mantuviesen, estariamos entrando en situaciones de hecho, abiertas a cualquier aventurerismo como el que supone el llamado Plan Ibarretxe».

Del análisis realizado del procedimiento agravado establecido en el artículo 168 de la Constitución española de 1978 cabe deducir, en primer lugar, que lo escueto de la regulación contenida en este precepto deja amplio margen a la interpretación o a la necesidad de un cierto desarrollo que, por otra parte, no realizan los Reglamentos de las Cámaras.

La regulación de la reforma contenida en el artículo X de la Constitución se caracteriza por establecer una pluralidad de sujetos y procedimientos que, en el caso del artículo 168, combina prácticamente todos los posibles obstáculos que ofrece el Derecho comparado (mayorías muy cualificadas, aprobación por dos legislaturas sucesivas, incluso dos veces en una de ellas, ratificación de la reforma en referéndum obligatorio).

2 G. Peces-Barba Martínez, «La reforma de la Constitución», Claves de razón práctica no 148, 2004, pág. 27. 
3. Las materias enumeradas en el artículo 168 de la Constitución para definir su ámbito de aplicación muestran, en primer lugar, que aquella admite su revisión total, a diferencia de otros ordenamientos de nuestro entorno, aunque sin especificar qué debe entenderse por ella (frente al Informe de la Ponencia constitucional, que atribuía su determinación al Tribunal Constitucional). Parece que debería ser una combinación de criterios cualitativos y cuantitativos la que llevara a calificar de total una reforma, aunque la determinación de que deben tramitarse por el procedimiento agravado las llamadas por Schmitt «decisiones constitucionales fundamentales» privaría de relevancia a esta disquisición. Serán, en todo caso, las Mesas de las Cámaras, en el momento de la calificación de las iniciativas, las que deberán resolver.

La Constitución no establece límites expresos a la reforma, sustituyéndolos por la extremada rigidez de algunas de sus partes, definidas de tal forma que cualquier retoque, por mínimo que sea, de uno de los artículos incluidos en las partes señaladas queda sujeto al procedimiento agravado. Tal es la opinión doctrinal mayoritaria, que parece compartir el Consejo de Estado, frente a aquellos autores que estiman posible restringir la aplicación del artículo 168 a las reformas que afecten a la totalidad de alguno de los Títulos en él reseñados.

El ámbito especialmente protegido coincide con los dos tipos de materias que otras Constituciones consideran irreformables: la forma política del Estado y los principios constitucionales básicos, a los que se añaden los derechos fundamentales y libertades públicas, que podrían considerarse también implícitos en los anteriores. No obstante, se ha criticado la exclusión de preceptos relevantes, como el 10, el 14 o el 53, o el propio Título X, y postulado la posibilidad de entender incluidas otras partes conectadas con las señaladas.

En cuanto a la posible existencia de límites implícitos, sin perjuicio de que algunos autores suelen coincidir en varios de ellos, como las propias normas de reforma constitucional o los derechos inviolables de la persona, la sentencia 48/2003 del Tribunal Constitucional es clara en cuanto a la inexistencia de un núcleo normativo inaccesible a los procedimientos de reforma constitucional.

4. La regulación de la reforma constitucional contenida en el Título X comienza en el artículo 166 con una remisión de la iniciativa al precepto regulador de la iniciativa legislativa, pero excluyendo la iniciativa popular.

Manteniendo el mismo criterio que respecto de la iniciativa legislativa, entendemos que dos son los titulares de la iniciativa de reforma constitucional, Gobierno y Parlamento, disponiendo los diputados, senadores y grupos parlamentarios, así como las Asambleas de las Comunidades Autónomas, de la facultad de propuesta, que deberá ser tomada en consideración por una de las Cámaras para 
convertirse en auténtica iniciativa. La exclusión de la iniciativa popular ha sido criticada por la doctrina. Tal vez con la perspectiva que da el tiempo transcurrido desde la aprobación de la Constitución y lo inoperante que se ha revelado una iniciativa legislativa muy restringida, la cuestión haya perdido algo de su importancia. En cambio, de haber sido admitida, podría pensarse en la posibilidad de que uno de los dos grandes partidos, que tienen abierta la iniciativa parlamentaria, pretendieran utilizar el peso político de una iniciativa popular de reforma constitucional.

El principal problema planteado en relación con la iniciativa de reforma constitucional es la forma que debe adoptar, tema relacionado con lo que deba entenderse por el principio de reforma que ha de ser aprobado por la primera legislatura. Sin que nada se desprenda del artículo 168, ni tampoco expresamente de los Reglamentos (salvo de un precepto de dudosa aplicación al procedimiento que nos ocupa en el Reglamento del Senado), entendemos, como también lo hace el Informe del Consejo de Estado, que la iniciativa de reforma agravada debe consistir en un texto escrito, no forzosamente articulado, que indique los artículos o partes de la Constitución a que aquella afectaría, así como el sentido de la reforma, es decir, en líneas generales, en qué va consistir o cuál es su finalidad, exigencia lógicamente derivada de que el conocimiento de las Cámaras que han de pronunciarse sobre el principio de reforma ha de ser pleno, sin perjuicio de que la orientación o finalidad de la misma sea expuesta de forma lo suficientemente amplia para que no coarte como límite la actuación de las segundas Cortes en la determinación del texto de la reforma.

5. Excluidas las cláusulas de intangibilidad, acoge la Constitución el otro tipo de límites habitualmente fijados al constituyente constituido, el temporal. La prohibición de la iniciativa de reforma, que afecta por igual al procedimiento simple y al agravado, alcanza a cuatro situaciones excepcionales o de anomalía constitucional. De ellas, las tres últimas, los estados de alarma, excepción y sitio, están perfectamente delimitadas, en cuanto a su declaración y su duración, en el artículo 116. Más dificultades presenta la definición de qué debe entenderse por tiempo de guerra. Una interpretación coherente de los artículos 169 y 63.3 llevaría a pensar que la limitación afecta sólo a los supuestos de guerra declarada formalmente. Pero hoy en día, con la flexibilidad con que se producen los conflictos bélicos, cabría pensar en situaciones de guerra no declarada en las que no procediera iniciar una reforma constitucional.

6. Con la presentación de la iniciativa de reforma constitucional en una de las Cámaras (el Congreso de los Diputados, si aquélla procede del Gobierno, de 
diputados o grupos de esta Cámara o de las Asambleas autonómicas; el Senado, si procede de los miembros o grupos de la Cámara Alta), entran en juego las facultades de calificación de escritos de la Mesa respectiva, previstas en los artículos 31.1.4 y $^{\circ}{ }^{\circ}$ del Reglamento del Congreso y 36.1.c) del Reglamento del Senado. La Mesa ha de verificar el cumplimiento de los requisitos de legitimación, distintos para cada tipo de iniciativa, así como de tiempo (inexistencia de la limitación temporal prevista en el artículo 169 de la Constitución) y de forma, debiendo quedar suficientemente identificadas las partes o preceptos constitucionales a los que afectará la reforma, pues sobre ellos pivotará la decisión de la Mesa en cuanto al procedimiento que ha de seguir aquélla.

Una vez admitida a trámite la iniciativa de reforma como tal, la decisión se centra en determinar si debe tramitarse por el procedimiento previsto en el artículo 167 o en el artículo 168 de la Constitución. Dado el carácter subsidiario o residual con que se ha configurado el primero, la decisión vendría determinada por la apreciación de que la iniciativa supone, en los términos del segundo, la revisión total de la Constitución o una parcial que afecte a las partes en él enumeradas. Serán, probablemente, elementos cuantitativos y cualitativos los manejados para entender que una revisión es total, de una parte; y, de otra, forzosamente habrán de estimarse como parciales que entran en el ámbito del artículo 168 cualesquiera propuestas de modificación de uno sólo de los artículos incluidos en las partes de la Constitución citadas en dicho precepto, como se ha expuesto al tratar de las materias afectadas por aquél.

La recalificación de una iniciativa, decidiendo su tramitación por un procedimiento de reforma constitucional distinto del planteado, es considerada admisible, partiendo de la doctrina del Tribunal Constitucional -últimamente contenida en el auto 85/2006- sobre la facultad de la Mesa de determinar el tipo de procedimiento parlamentario sin mutar a otros previstos para iniciativas de naturaleza distinta. Para analizar los distintos supuestos posibles de recalificación, cabe aplicar la analogía con la regulación y la práctica existentes respecto de las leyes orgánicas.

En primer lugar, ha de afirmarse que la decisión sobre si la iniciativa es única o múltiple corresponde a su autor, facultado para presentar, a su discreción, uno o varios proyectos o proposiciones de reforma, que, en buena técnica normativa, deberían estar diferenciados por su procedimiento de tramitación.

Si se optara por una iniciativa única y esta afectara a materias susceptibles de tramitación por ambos procedimientos, no habría razones para que la Mesa recalificara aquélla siempre que se presentara para su tramitación por el procedimiento agravado, porque no violaría las reglas constitucionales de rigidez el aplicar dicho procedimiento a materias que no lo requieren. En el supuesto contrario, 
esto es, de presentación de una iniciativa mixta de reforma para su tramitación conforme al artículo 167 de la Constitución, la Mesa no podría admitir este procedimiento y, de seguir un criterio similar al observado respecto de las leyes orgánicas, procedería al desglose de aquellas partes de la iniciativa que han de tramitarse por el procedimiento agravado, siempre que no afectaran al núcleo de la misma (por utilizar la terminología empleada por el Tribunal Constitucional respecto de las leyes orgánicas en la sentencia 76/1993), pues en tal supuesto toda la iniciativa en bloque habría de ser tramitada por el procedimiento agravado.

7. La remisión que contiene el artículo 166 de la Constitución al 87.1 y 2, en cuanto al ejercicio de la iniciativa legislativa, y a su vez la segunda remisión contenida en dicho precepto a los Reglamentos de las Cámaras, reforzada por la expresa declaración del artículo 89 de que la tramitación de las proposiciones de ley se regulará por ellos, abonan la interpretación de que las proposiciones de reforma, como las de iniciativa legislativa, han de someterse al trámite de toma en consideración.

Desde un punto de vista puramente procedimental podría parecer que sólo deben ser objeto de toma en consideración las proposiciones de reforma ordinaria de la Constitución (como ocurrió en la reforma del artículo 13.2), que han de ser articuladas y se tramitan en esa legislatura hasta aprobar en su caso, la reforma constitucional completa. Las proposiciones de reforma agravada, en cambio, que podrían no ser articuladas y sólo son objeto en la primera legislatura de debate y votación de totalidad, podrían ser objeto de una sola decisión en este debate, siguiendo al pie de la letra lo dispuesto en el artículo 147 del Reglamento del Congreso.

Sin embargo, otras consideraciones más de fondo y relacionadas con trámites posteriores, nos inclinan hacia la solución contraria. Si la finalidad de los procedimientos parlamentarios, y del legislativo en particular, es permitir la participación de las distintas fuerzas políticas representadas en el ejercicio de las funciones de las Cámaras y tratar de obtener el mayor consenso posible en las decisiones de éstas, tiene sentido que exista un primer pronunciamiento liminar sobre las proposiciones de reforma, que permita después modificarlas -aun manteniendo su naturaleza genérica de principio en el caso de las reformas agravadas- para que la eventual aprobación del principio de revisión alcance la mayor unanimidad posible. En consecuencia, parece que procede la sumisión de las proposiciones de reforma agravada, como las que postulan la simple, al trámite de toma en consideración por el Congreso de los Diputados.

La emisión de criterio por el Gobierno resulta de la aplicación del artículo 126.2 del Reglamento del Congreso. Este trámite, cuyo sentido en la reforma 
constitucional es dudoso, fue no obstante observado en la reforma de 1992 e incluso el Gobierno formuló su criterio expresamente para no demorar el procedimiento.

8. Aunque tanto la regulación constitucional (arts. 166 y 168) como la reglamentaria (arts. 146.1 y 147 RC, $152-153$ y 158 RS) pasan directamente de contemplar la iniciativa a la votación del principio de reforma por el Pleno, nos hemos planteado si debiera abrirse un trámite de enmiendas antes de someter el proyecto o proposición de reforma a la votación del «principio de revisión».

Algunas razones nos inclinan a considerar razonable la apertura de un plazo de enmiendas en el Congreso. Las enmiendas permiten mejorar un texto, incorporar a su elaboración a las fuerzas políticas representadas en la Cámara -si la propuesta procede del Gobierno- o a las no firmantes, y ampliar el ámbito de consenso sobre la misma, lo cual es particularmente deseable en la aprobación de un principio de reforma constitucional, no sólo por las mayorías exigidas, sino por su significado.

Es claro que, ante el silencio reglamentario, habría de ser una Resolución de la Presidencia del Congreso de los Diputados, adoptada en la forma establecida por el artículo 31.2 del Reglamento, esto es, previo parecer favorable de la Mesa y de la Junta de Portavoces, la que estableciera el trámite y sus límites. Pero cabe pensar que sería adecuada una regulación parecida a la prevista en el Reglamento para una iniciativa no legislativa, las proposiciones no de ley. No en vano hemos admitido que una propuesta de reforma agravada no sea articulada y, en todo caso, lo que se votaría sería el principio de la reforma, incorporando, en su caso, las enmiendas aceptadas por el autor de la iniciativa.

9. El debate sobre el principio de la reforma, como debate de totalidad, habrá de versar sobre la oportunidad, principios o espíritu de aquella, o, dicho de otra manera, sobre la conveniencia, ámbito y orientación de la misma, expresados en un documento no forzosamente articulado, incluso de preferencia un documento político no legislativo.

El efecto más importante de la aprobación por el Congreso es que queda fijado el principio de reforma, que ha de ser aprobado por el Senado en los mismos términos para que el procedimiento continúe, de una parte; y que deberá ser ratificado también en los mismos términos por las nuevas Cámaras elegidas, de otra parte, conforme al artículo 168.2 de la Constitución.

10. Recibido en el Senado el principio de reforma, ya proceda de una proposición tomada en consideración por esta Cámara y tramitada en el Congreso, 
ya de cualquier otro titular de la iniciativa (aunque el artículo 158.1 sólo cita «el proyecto recibido del Congreso o la proposición presentada en el Senado"), por disposición de este artículo serán elevados directamente al Pleno. Queda claro, pues, que no cabe trámite de enmiendas respecto del principio de reforma en el Senado, lo cual no es contradictorio con lo anteriormente defendido para el Congreso, sino muy coherente con la regulación constitucional, que no prevé (como tampoco para decisiones ulteriores) solución de discrepancias entre las Cámaras en cuanto a la decisión sobre el principio de reforma, por lo que en este caso parece claro que, salvo que se admita que el Senado puede tramitar sus iniciativas de reforma agravada en primer término, su labor en este momento se ve reducida a ratificar o no la decisión adoptada en el Congreso.

El efecto principal de la aprobación del principio de reforma por mayoría de dos tercios en el Senado es que la iniciativa de reforma agravada ha sido aprobada por ambas Cámaras y, en consecuencia, ha prosperado, continuando el procedimiento previsto en el artículo 168 de la Constitución mediante la «disolución inmediata de las Cortes».

La no obtención en el Senado de la mayoría requerida de dos tercios supone el fracaso de la iniciativa de reforma, que concluye su tramitación pese a la mayoría obtenida en el Congreso. No prevé el artículo 168 de la Constitución solución alguna para tal discrepancia de las Cámaras. De forma excepcional, no se impone la voluntad del Congreso, sino que ambas Cámaras están en situación de paridad, como ocurre sólo en otro supuesto, con el que cabe trazar algún paralelismo procedimental: la exigencia por el artículo 150.3 de la Constitución de la mayoría absoluta de cada Cámara para la aprobación de la necesidad de dictar leyes armonizadoras, supuesto de discrepancia también inédito en nuestros años de vigencia de la Constitución.

11. La Constitución ordena (mediante la utilización del tiempo futuro) que se proceda a la disolución inmediata de las Cortes. Por mucho que se la califique de ope legis o automática en la doctrina, ha de llevarse a cabo por los procedimientos establecidos, esto es, mediante un Real Decreto firmado y refrendado, que entrará en vigor una vez publicado en el Boletín Oficial del Estado, aunque sea el mismo día, como dispone el artículo 42 de la Ley Orgánica del Régimen Electoral General para los decretos de convocatoria en caso de disolución anticipada.

La disolución ha de ser inmediata, pero debe seguir un cauce jurídico, por lo que no surtirá efectos, al menos, hasta el día siguiente, si el Real Decreto se expide el mismo día de la aprobación del principio y se publica al día siguiente. No creemos que sean inválidas las actuaciones de las Cámaras producidas el mis- 
mo día de aprobación del principio de reforma y hasta la entrada en vigor del Real Decreto de disolución, como no lo son en el caso de elecciones anticipadas las que realizan el día de su expedición, inmediato anterior al de su entrada en vigor.

No cabe duda de que las elecciones convocadas poseen un carácter especial que las diferencia de otras elecciones generales. El electorado, al pronunciarse sobre las distintas opciones políticas que ofrecen las diferentes candidaturas concurrentes a las elecciones, emite también su criterio (o, al menos, tal finalidad parece implícita en la disolución impuesta) sobre la reforma constitucional en curso. Pero no estimamos, sin embargo, que prevalezca en estas elecciones el significado de consulta popular o que tengan una finalidad diferente de lo que son los comicios electorales generales.

Es indiscutible que la reforma constitucional, y hasta cierto punto la orientación de la misma dentro de los límites aprobados en el principio de reforma, formará parte de la campaña electoral. Pero, precisamente, su aprobación por mayoría de dos tercios permite concebir que las fuerzas políticas mayoritarias en contienda mantienen un acuerdo básico sobre la reforma, aunque existan diferencias de matiz. La experiencia de estos años ha demostrado que cuando se piensa en una reforma agravada de la Constitución se pretende hacer coincidir la disolución prescrita con la ordinaria por expiración de la legislatura (o, a los mismos efectos, con una disolución anticipada técnica, próximo el fin de aquélla), con lo que la campaña electoral no se centrará fundamentalmente en la reforma constitucional, sino en el programa político de los partidos para la siguiente legislatura.

Se ha definido en general por la doctrina a las Cámaras de la segunda legislatura como constituyentes. No cabe duda de que lo serán, en el sentido de que tienen encomendada la reforma de la Constitución respecto de la que se ha aprobado el principio, que puede ser incluso una reforma total, pero ello no excluye su actividad de órgano legislativo ordinario. Se trata, creemos, más bien de unas Cortes ordinarias (que no tendrán que disolverse cuando culminen la reforma de la Constitución) que tienen encomendada una tarea constituyente, para la que han sido elegidas en primer lugar. Esto último será más evidente si la disolución fue ex professo para la reforma constitucional, que si se hizo coincidir con la finalización de una legislatura.

12. Entendemos que las segundas Cortes se encuentran vinculadas por el principio aprobado, que acota en mayor o menor grado, según la amplitud con que esté formulado, el ámbito de actuación de la segunda legislatura. Esta vinculación opera en un doble sentido: en primer lugar, la decisión a ratificar se 
materializa en la sumisión a votación del mismo texto o iniciativa aprobada en la primera legislatura; en segundo lugar, en la elaboración posterior del texto, esa iniciativa o principio aprobado en la legislatura anterior actúa como límite para las Cortes.

En otro caso, si se excediera de los artículos o partes señaladas en la iniciativa y se reformaran otras no indicadas en ella, no se respetaría respecto de éstas el procedimiento establecido en el artículo 168 de la Constitución, pues, en cuanto a ellas, no habría recaído aprobación respecto del principio de reforma, ni habría habido disolución y elecciones posteriores.

La Constitución no requiere mayoría cualificada alguna para la ratificación de la decisión de reforma. Tampoco la exige el Reglamento del Congreso, pero sí el artículo 159 del Reglamento del Senado, según el cual, «la nueva Cámara que resulte elegida deberá ratificar, por mayoría absoluta de sus miembros, la reforma propuesta». La doctrina se ha planteado la inconstitucionalidad de este precepto, en la medida en que el artículo 79.2 de la Constitución sólo permite a los Reglamentos de las Cámaras (junto a la propia Constitución y a las leyes orgánicas) establecer mayorías especiales para la elección de personas.

Desde el punto de vista jurídico, podrían plantearse situaciones problemáticas si no se obtuviera mayoría absoluta en el Senado y se declarara adoptado el acuerdo. A la inversa, podría estimarse no alcanzado el acuerdo obteniendo mayoría simple en el Senado, por aplicación del artículo 159.2 de su Reglamento. Todo ello nos lleva a propugnar, como solución más sencilla, la reforma del Reglamento del Senado en este punto, para acomodarlo a la Constitución.

No prevé el artículo 168 procedimiento alguno para solventar la discrepancia entre Congreso y Senado en este momento, como tampoco más adelante. La ratificación de la reforma requiere el acuerdo concurrente de ambas Cámaras, como ocurría ya en las primeras Cortes con la aprobación del principio de reforma. Ello forma parte del cúmulo de obstáculos que ha ideado el constituyente para dificultar la reforma agravada. No creemos que sea éste el momento procesal que permita plantearse, extra Constitutione, si cabe concebir alguna forma de dirimir el conflicto o, más propiamente, de eliminar el obstáculo. Una de las Cámaras surgida de las elecciones convocadas a este efecto estima que no procede seguir adelante con la reforma. Hasta aquí ha llegado ésta, la iniciativa ha fracasado porque su voluntad de no continuar no puede ser suplida ni cabe requerir un nuevo pronunciamiento.

13. La función que el artículo 168 de la Constitución atribuye a las nuevas Cámaras, una vez ratificada la decisión de reforma, consiste en proceder al estudio del nuevo texto constitucional. Estos términos, que aparecen ya literales en el 
informe de la ponencia constitucional, no han dejado de llamar la atención de la doctrina, pues de su interpretación depende en parte la estructura de todo el procedimiento de reforma. Dada la concepción que hemos adoptado de la iniciativa de la reforma, partimos de que las nuevas Cortes carecen de un texto articulado que comenzar a tramitar por el procedimiento legislativo común. Se hace preciso, por tanto, elaborar un texto que cumpla las funciones de primer borrador que en el procedimiento legislativo desempeña un proyecto o proposición de ley.

En cuanto a la elaboración de este texto o anteproyecto, en general, la doctrina parece inclinarse por una elaboración "parlamentaria». Opinamos que esto es sólo una opción, como la adoptada en 1977 para el primer borrador de la Constitución. En el momento actual, siempre que no se trate de una reforma total, no habría razones para excluir el origen gubernamental del nuevo texto; no repugna al procedimiento diseñado por el artículo 168 de la Constitución que el Gobierno remitiera en la segunda legislatura un proyecto articulado de reforma al Congreso de los Diputados, una vez ratificada la decisión de reformar por ambas Cámaras.

Tampoco carecería de lógica que los grupos parlamentarios, resultantes de unas elecciones "constituyentes», presentaran una o varias proposiciones articuladas de reforma, siempre dentro de los límites marcados por el principio aprobado en la anterior legislatura.

La tercera posibilidad sería encomendar la elaboración del texto a una ponencia en el seno de la Comisión Constitucional del Congreso, esto es, al conjunto de las fuerzas políticas representadas en la Cámara.

Aunque cabe admitir la posibilidad de que cualquiera de las Cámaras inicie la tramitación de la reforma, lo que podría clarificar el nonato Reglamento de las Cortes Generales, su inexistencia nos lleva a optar por seguir las reglas previstas para el procedimiento legislativo en la propia Constitución, iniciando la tramitación del texto en el Congreso de los Diputados.

Dos cuestiones se nos suscitan relacionadas con el trámite de enmiendas y aplicables también al procedimiento que ha de seguirse en la Cámara Alta: en primer lugar, parece que debiera queda excluida la presentación de enmiendas a la totalidad de devolución (propuestas de veto, en el Senado), porque ha existido ya un pronunciamiento previo favorable a la tramitación, al ser ratificada la decisión de reforma. Es cierto que, en ese momento, el nuevo texto no tenía por qué existir, si no fue presentado articulado en la anterior legislatura, pero introducir un tercer debate y votación de totalidad no parece que estuviera en la voluntad del constituyente y sólo sería admisible para permitir la aprobación de enmiendas de texto alternativo. 
En segundo lugar, parece imprescindible exigir la homogeneidad y congruencia de las enmiendas con el texto elaborado o, al menos, con el principio de reforma ratificado. Pese a la oscilante doctrina del Tribunal Constitucional en cuanto a la necesidad o no de conexión material de las enmiendas con el texto legislativo enmendado, aquí más que nunca debemos defender que han de observarse estos requisitos, para que por vía de enmienda no se introduzcan modificaciones no previstas en el principio de reforma aprobado por la primera legislatura y ratificado por la segunda, que esquivaran las reglas de procedimiento establecidas por el artículo 168 de la Constitución.

14. La tramitación del texto de reforma por el Congreso de los Diputados concluye con su debate y aprobación por el Pleno, para la que el artículo 168 de la Constitución exige mayoría de dos tercios. Estimamos que esta exigencia debe aplicarse a una votación final sobre el conjunto del texto resultante de las diferentes votaciones, como prescribe el artículo 81 de la Constitución para las leyes orgánicas.

Una votación similar deberá producirse al término del debate y votación del texto en el Senado (como determina el artículo 159 de su Reglamento), debiendo alcanzar idéntica mayoría sobre el mismo texto para que la reforma constitucional se entienda aprobada por las Cámaras.

Uno de los problemas más complejos de interpretación que presenta el artículo 168 de la Constitución, deriva de que este precepto no prevé la solución de discrepancias entre las Cámaras. Ello podría llevar a pensar, en una primera aproximación, que se trata de una dificultad adicional introducida y que, si no recae acuerdo de ambas Cámaras, la reforma queda desechada. Pero puede ocurrir que el Senado apruebe, también por mayoría de dos tercios, un texto distinto del que aprobó el Congreso de los Diputados, por haber incorporado enmiendas al mismo. Tal sería el resultado más lógico de la paridad de las Cámaras en el procedimiento.

Si se admitiera que la aprobación por mayoría de dos tercios por las Cámaras de dos textos diferentes supone el fracaso de la reforma, la segunda Cámara que intervenga en la tramitación del texto vería limitado su papel, si no quiere hacer fracasar la reforma, a ratificar el texto aprobado por la otra, conclusión a todas luces absurda. Si ambas Cámaras se hallan en situación de paridad, ambas pueden utilizar su facultad de enmienda y, en consecuencia, aprobar textos distintos. Alguna solución habrá de arbitrarse para permitir que llegue a alcanzarse el acuerdo de ambas Cámaras por mayoría de dos tercios sobre un mismo texto, dando por supuesto que esta fase conciliadora queda excluida si una de las Cámaras no ha aprobado el texto de reforma por mayoría de dos tercios, como exige la Constitución. 
Esta posibilidad, creemos, no fue contemplada por el constituyente, ni en consecuencia regulada. No cabe acudir a la Constitución para darle solución. Habrá de ser otra norma, de preferencia el inexistente Reglamento de las Cortes Generales previsto en el artículo 72.2 de la Norma fundamental, el que colme la laguna normativa y establezca un procedimiento para salir de un impasse no previsto en el artículo 168, permitiendo llegar al resultado exigido por este precepto para la aprobación de la reforma. En defecto de este Reglamento, los de cada una de las Cámaras o una norma adoptada por las Mesas del Congreso de los Diputados y del Senado, en reunión conjunta, pueden prever un procedimiento a estos efectos.

Tal vez lo más adecuado fuera encomendar la redacción de un texto de acuerdo a una Comisión mixta paritaria, como opinan algunos autores. Pero nunca sería posible la aplicación analógica del artículo 167.2 de la Constitución en cuanto a la forma última de aprobación de dicho texto, es decir, en cuanto a la posibilidad de aprobación de la reforma, siempre que hubiera obtenido el voto favorable de la mayoría absoluta del Senado, por mayoría de dos tercios del Congreso de los Diputados. De establecerse el recurso a la Comisión mixta paritaria por una norma de las antes señaladas, el texto elaborado por ella habría de obtener mayoría de dos tercios en una votación de conjunto en cada una de las Cámaras, para que la reforma fuera aprobada.

Nada impide que una norma permita repetir el intento de conciliación hasta obtener las mayorías exigidas, aunque cada vez nos alejamos más del espíritu de rigidez que inspira el artículo 168 de la Constitución, forzando un poco más su primera interpretación literal.

En cuanto al otro procedimiento posible, el de navette del texto entre las Cámaras hasta alcanzar un texto de consenso que obtenga las mayorías requeridas, parece que, al menos, sería admisible que -incluso sin norma habilitante- el Presidente del Senado (si esta es la segunda Cámara que interviene en la tramitación) remitiera al del Congreso el texto aprobado por mayoría de dos tercios, y que éste fuera sometido a votación de conjunto en esta Cámara, para ver si obtiene la misma mayoría, en una suerte de analogía con el procedimiento de aprobación de las leyes orgánicas. Esta primera navette no parece plantear mayores problemas, pero de nuevo se fuerza más la interpretación del artículo 168 de la Constitución a medida que se acepta que se repita el reenvío entre las Cámaras.

Admitiendo estas posibilidades, nos situamos fuera del artículo 168 de la Constitución (no contra ella), por considerar necesario resolver una situación no prevista por la misma, sin dar al traste con una reforma constitucional que ha superado graves obstáculos hasta llegar a este momento próximo del final, y que cabe entender no ha fracasado por no obtención de las mayorías requeridas. 
Cualquiera que sea la solución adoptada, el resultado final ha de ser el mismo: un texto de reforma único aprobado por mayoría de dos tercios en cada una de las Cámaras. Otro modo de culminar la reforma no es constitucionalmente posible.

15. El último obstáculo que debe salvar la reforma tramitada conforme al artículo 168 de la Constitución es su ratificación en referéndum. La doctrina destaca la contradicción que supone incluir en un mismo procedimiento de reforma dos consultas populares, las elecciones intermedias y el referéndum final, añadidas además a un sistema procesal muy complejo. En este sentido, se ha calificado al referéndum de redundante. No obstante, creemos acertada la inclusión de un referéndum final de ratificación de la reforma, porque da al electorado la posibilidad de pronunciarse sobre el resultado definitivo de la misma.

La segunda circunstancia destacada es que la Constitución no requiere para este referéndum quórum alguno de votantes, ni tampoco de votos afirmativos. Basta con que el número de éstos supere a los negativos, cualquiera que sea el número de votantes, para que la reforma quede ratificada.

16. Una vez ratificada por el pueblo la reforma aprobada por las Cortes Generales, ha de procederse a su promulgación por el Rey y a su publicación el Boletín Oficial del Estado. Pese al precedente de la reforma de 1992, la cual fue sancionada por el Rey, consideramos que no debe ser objeto de sanción ninguna reforma constitucional, haya intervenido o no el pueblo en su aprobación. En ambos casos, por haber participado el poder constituyente constituido, del que no forma parte el Rey, al que la Constitución atribuye sólo la sanción de las leyes aprobadas por las Cortes Generales en los artículos 62 a) y 91, y cuyas funciones limita el artículo 56 a las que le atribuyen expresamente la Constitución y las leyes.

17. Además de por su aprobación o su rechazo en alguna de las fases del procedimiento, la reforma constitucional puede concluir por otras dos formas de terminación, la retirada y la caducidad.

No existiendo previsión alguna constitucional o reglamentaria, entendemos aplicables a la retirada de las iniciativas de reforma constitucional las normas relativas al procedimiento legislativo. Corresponde la facultad de retirada de los proyectos de reforma al Gobierno, que podrá retirarlos en la legislatura en que los presentó, en cualquier momento anterior a que las dos Cámaras se hubieran pronunciado sobre ellos, obteniendo la mayoría de dos tercios que supone la inmediata disolución. Sin embargo, una vez aprobado el principio de reforma, ni 
antes de la disolución (por haberse pronunciado ya las primeras Cámaras definitivamente sobre el mismo) ni después de ésta cabe la retirada de la propuesta. Aplicando por analogía el régimen de las proposiciones de ley, las proposiciones de reforma parlamentarias o autonómicas pueden ser retiradas antes de su toma en consideración por la Cámara ante la que se presentan; con posterioridad a este trámite, el autor de la proposición podrá presentar la iniciativa de retirada, que para ser efectiva precisará del acuerdo de la Cámara que la tomó en consideración.

La aplicación analógica del régimen de caducidad de las iniciativas legislativas a las propuestas de reforma conduce a entender que la conclusión de la primera legislatura de la reforma por expiración del mandato o por disolución anticipada supone la caducidad de las propuestas de reforma en trámite presentadas por el Gobierno o por sujetos parlamentarios, hayan sido estas últimas tomadas o no en consideración. Siempre, obviamente, que no hayan alcanzado la mayoría requerida en las dos Cámaras que provoca la disolución. En cuanto a las propuestas de reforma procedentes de Asambleas autonómicas, la aplicación del régimen de las proposiciones de ley supondría la no caducidad de las propuestas no sometidas a la toma en consideración, que serían trasladadas a la siguiente legislatura, mientras que caducarían aquellas que hubieran sido tomadas en consideración y se hallaran pendientes de aprobación por las Cámaras, porque ya fueron asumidas por el Congreso de los Diputados.

En la segunda legislatura, el principio de reforma caduca si no llega a ser sometido a ratificación, lo que podría llegar a acontecer si se produce una disolución automática por aplicación del artículo 99.5 de la Constitución, en cuyo caso entendemos que con la segunda disolución habrá caducado la propuesta de reforma presentada en la primera legislatura y aprobada por las primeras Cortes, debiendo iniciarse el procedimiento nuevamente, si se desea llevarlo a término, con la presentación y aprobación de una nueva propuesta.

La reforma constitucional aprobada por las dos Cámaras y pendiente de referéndum no caducaría por la disolución, por faltar sólo la concurrencia del acto de otro sujeto. La celebración del referéndum podría incluso coincidir con las elecciones convocadas, dada la excepción a la prohibición general contenida en la Ley Orgánica 2/1980.

18. Las críticas formuladas al artículo 168 de la Constitución han conducido a la doctrina a plantearse la cuestión de su reforma. En concreto, se trata de si este artículo, no incluido entre las partes de la Constitución sujetas al procedimiento agravado de reforma, ha de entenderse implícitamente contenido entre ellas o, más aún, si debe considerarse un límite implícito de la Constitución 
y, en consecuencia irreformable. En cuanto a esto último, creemos que, si la Constitución ha previsto y permitido su revisión total, ninguna de sus partes queda exenta de la posibilidad de reforma, siempre con sujeción a los procedimientos establecidos.

Por lo que se refiere a la posibilidad de modificar el artículo 168 por la vía del artículo 167, entendemos que son dos los argumentos contrapuestos que han de sopesarse para tratar de llegar a una conclusión. En primer lugar, la no referencia al artículo 168 entre las materias por él enumeradas como dotadas de especial rigidez permitiría entenderlo excluido de su ámbito y, en consecuencia, reformable por la vía del artículo 167; pero, de otra parte, no deja de latir en el fondo de tal razonamiento la impresión difusa de que se estaría yendo contra la voluntad del constituyente, por no emplear la expresión más fuerte de fraude a la Constitución. Si se utiliza un enfoque literalista y formal, estrictamente jurídico, la solución se inclina en favor del primer criterio; sólo si se añaden ingredientes de índole metajurídica caería el peso de la balanza hacia el segundo.

19. Una última cuestión planteada en relación con el procedimiento de reforma constitucional agravada es si es susceptible de control por el Tribunal Constitucional y por qué vía. En abstracto, es evidente que de la mera existencia de dos procedimientos de reforma diferenciados por la materia (como ocurre cuando se establecen cláusulas de intangibilidad) deriva la necesidad de un control que verifique su correcta utilización, como también la sumisión a los límites establecidos, en nuestro caso sólo temporales. Pero de lege lata, ni la Constitución ha dispuesto mecanismo alguno de garantía de los preceptos reguladores de su reforma ni la Ley Orgánica del Tribunal Constitucional, que pudo hacerlo, lo ha hecho.

Frente a la opinión que admite someter las reformas constitucionales (sean o no consideradas leyes) al recurso de inconstitucionalidad, creemos que es posible abordar el problema de la vía jurisdiccional desde otra perspectiva. Siendo lo más adecuado una reforma de la LOTC que incluyera entre los actos impugnables las reformas de la Constitución (de preferencia en recurso previo anterior a su ratificación en referéndum), en su ausencia, para asegurarse del cumplimiento de las normas constitucionales que distinguen dos procedimientos, lo procedente sería ejercer otras posibilidades de control sobre las etapas intermedias del proceso de gestación de la reforma (aunque la doctrina contenida en algunas decisiones del Tribunal Constitucional pudiera dificultar aquél, el propio ATC 135/2004 reconoce la relevancia de la eventual inconstitucionalidad de los actos de trámite cuando "producen una lesión inmediata de los derechos fundamentales de los sujetos legitimados para participar en el procedimiento»), en particular en relación con la 
decisión sobre el procedimiento de tramitación que corresponde realizar a la Mesa de la Cámara en ejercicio de su función calificadora de las iniciativas presentadas, que podría ser susceptible de un recurso de amparo por infracción del artículo 23, interpuesto por alguno de los miembros de las Cámaras que considerara violado su derecho al procedimiento reconocido por la STC 99/1987.

Title

The increased procedure of reform of the Constitution of 1978

\section{Resumen}

En este trabajo se lleva a cabo un pormenorizado análisis del procedimiento agravado de Reforma constitucional, regulado en el art. 168 de la Constitución española. Se abordan los posibles problemas a que puede dar lugar el procedimiento, especialmente en el ámbito parlamentario, y se proponen algunas soluciones a dichos problemas.

\section{Abstract}

This work makes an itemized analysis of the increased procedure of constitutional Reform, regulated in the art. 168 of the Spanish Constitution. The possible problems are approached to that can give place the procedure, especially in the parliamentary environment, and the author intends some solutions to this problems.

\section{Palabras clave}

Reforma constitucional, Procedimiento de reforma constitucional, control de la reforma constitucional

\section{Key words}

Constitutional reform, Procedure of constitutional reform, control of the constitutional reform 